\title{
Study and Application of Comprehensive Analysis System on Reservoir Engineering
}

\author{
Yao-Zhong Yang \\ Information management division, \\ Shengli oilfield, Sinopec, \\ Dongying city, Shandong Province, 257001 \\ E-mail: yangyz2058@sina.com
}

\author{
Ye-Heng Sun, Guang-lai Yue, Tao Dai \\ Geoscience Research Institute, \\ Shengli oilfield, Sinopec, \\ Dongying city, Shandong Province, 257015 \\ E-mail: sunyh.slyt@sinopec.com
}

\begin{abstract}
The reservoir engineering is a highly comprehensive discipline used in the oilfield development, which is dependent on the petroleum geology, reservoir description, reservoir physics, well testing and well production, so as to plan and program the oilfield development, and direct the scientific and rational development of oilfield. The reservoir engineering proposed herein is based on WINDOWS programming, control components programming, parallel management coupling public database and module database, so as to realize the connection between development database and opening module programming, and therefore covering the whole process from reservoir features analysis, development technical limit analysis, development performance analysis, development forecast, economic evaluation, and utilities. This software is well adaptable to modern reservoir engineering management, and fully taking advantage of the computer in the field to avoid the abundant and repetitive calculation for the reservoir engineers. It is capable of enhancing the working efficiency, as well as analysis and management level.
\end{abstract}

Keywords-reservoir engineering; system; design; application

\section{INTRODUCTION}

The reservoir engineering is complex in terms of research content, multiple disciplines, and related working objects. Except the related reservoir petrophysical properties, flow mechanisms, and reservoir evaluation methods, only the conventional and classic analysis methods are highly complex, let along the recently developed heavy oil thermal flooding, tertiary recovery, and horizontal well and related numerical simulation. The classic and conventional methods can be referenced from the professional books, however, the new theory, methods and advances that are recently published are not collected systematically, and even less programmed in the computer system.

This reservoir engineering system is adaptable to the modern research and management requirement, and avoids the abundant and repetitive calculation for the reservoir engineers from daily work, so as to enhance the working efficiency, analysis and management level. It is based on the development database, and characterized by complete reservoir engineering methods, numerous functions, visualized interface, variable input and output selection, convenient and practical.

\section{KEY TECHNICAL STUDIES ON INTEGRATED RESERVOIR ENGINEERING SYSTEM}

The reservoir engineering methods are complex, flexible, subjective, and highly dependent on actual conditions. Therefore, the key to the system is the analysis of technical difficulties to the software, and the critical techniques should be resolved.

\section{A. Technical Difficulties}

During the study process, it is inevitable to encounter variable problems, and they are discussed as follows, so as to solve them for the realization of function.

- Consistence of development database: in order for the management of database and reduction of information redundance, this system is connected with 47 data sheet by means of key words, ensuring the uniqueness of the key words in every data sheet is of vital importance, and also the precondition for the application of database.

- Optimization of reservoir engineering methods: the methods used in the reservoir engineering are commonly generalized in the development process, and every method is only applicable under certain conditions, and different conditions may induce contrary results. Therefore, it is very important to meet the requirement of different researchers during the programming of the software.

- Interaction between data and image: the data in the database may change in accordance to the time and space, so, it is necessary to visualize the data changing in the temporal and spatial domain. Therefore, many data are visualized in form of curve and contour (Figure 1).

\section{B. Key Techniques Study}

In order to meet the requirement, the software system should cover 6 aspects of reservoir engineering, including reservoir basic feature analysis, development technical limit analysis, development performance analysis, development forecast, development evaluation, so as to meet the daily working requirement, and the key is as follows:

- Connection with development database: data is input from development database and creating the seamless connection with the database, the development database is fully utilized to enhance the working efficiency.

- Collection and filtering of reservoir engineering methods: there are more than 400 reservoir engineering methods collected that are suitable for different reservoir, development mode, and 
development stage, and 210 methods are selected and integrated in the software.

- Adaptability study on reservoir engineering methods: the reservoir engineering methods are complex and generalized by different expert from different point of view for different objects as well as different conditions. Therefore, it is important to filter the adaptable methods according to application conditions, and accommodate the reservoir conditions and development stage, and convenient for the user to utilize and select. For example, there are 13 water flooding methods for sand reservoir, among which, Sipachev curve is suitable for the whole flooding process, Sazonov curve not suitable for high water cut stage, Nazarov curve suitable for high and medium water cut stage but not for low water cut stage, and Tong curve suitable for medium water cut stage but not for low and high water cut stage. Generally, due to the high viscosity, low sweeping efficiency, high heterogeneity, coning and reservoir wettability, the low water cut stage is short and quickly enters into the high water cut stage which is responsible for most of the oil production, therefore causing the concave curve, and concave curve for the light oil, high permeable and hydrophile reservoir. And, the $\mathrm{S}$ shape curve is between concave and convex curve, and commonly attributed to the medium viscosity oil.

- These curves above are applicable under the condition that abundant production information and data are accumulated, and may be used for reserve estimation and recovery factor ascertaining which have proven feasible for the oilfield with certain water cut. According to the reservoir engineering study process, it may be classified into 5 groups, that is, basic reservoir features analysis, reservoir development technical limit study, reservoir development performance analysis, reservoir development forecast, reservoir development economic evaluation.

- Imaging: Two type of imaging are realized herein. The curve type, it can illustrate the indexes, such as stage oil production, accumulated oil production, stage water yielding, accumulated water yielding, composite water cut, change with the time, therefore making the understanding on well and block development rule more evident and visual. The contour type, it can draw the micro structure map, isopach map of sand, net pay map, porosity and permeability map.

\section{DETAILED DESIGN OF ANALYSIS SYSTEM ON RESERVOIR ENGINEERING}

Based on the previous study, it is necessary to realize the seamless connection with the database, and tasks such as:

- high data process capacity, capable of not only downloading the file from the development database, but also calling the prepared database or transferring the text file, and even constructing new database in the software;

- Calculation and imaging: it is of conventional functions such as geological and reservoir engineering parameters calculation, as well as development index forecast. Moreover, the contour map, histogram and other types of curves;

- It is of project management function: it can integrate the professional raw data with the result data into one platform; it is favorable for the data enquiry, supplement and perfection, and tracking service

\section{A. Software Structure}

The integration of software is managed as project, and listed as project catalogue (Fig 1). The data may be obtained from the field development database, and programmed in the Windows environment with visualization tools, and realized the control by means of button. The integrity of software adopts the expandable module structure, including three orders of menu, among which, 8 first class, 57 second class and 46 third class, totally 95, and more than 210 methods[1][2].

In the first class menu, it is mainly consisted of project database and the imported data from ORACLE, basic reservoir features analysis, reservoir development technical limit analysis, reservoir development performance analysis, performance evaluation, utility tools, and parameters setting.

\section{B. Data Structure}

The data of the software system is imported from the development database, local dynamic database, local user input data, which are all first imported into the public database, and module is responsible for corresponding data which is accessible to the user's modification, and the module data may provide all the data used for the calculation done by each module.

In the process of realization of this function, each data in each module should be analyzed in detail including data source, data flow, storage position and storage mode. One calculation module may need many data, and the public data may be searched from the public database by means of specific data type and corresponding data table; if not public data, it must be input or imported from the module database. 


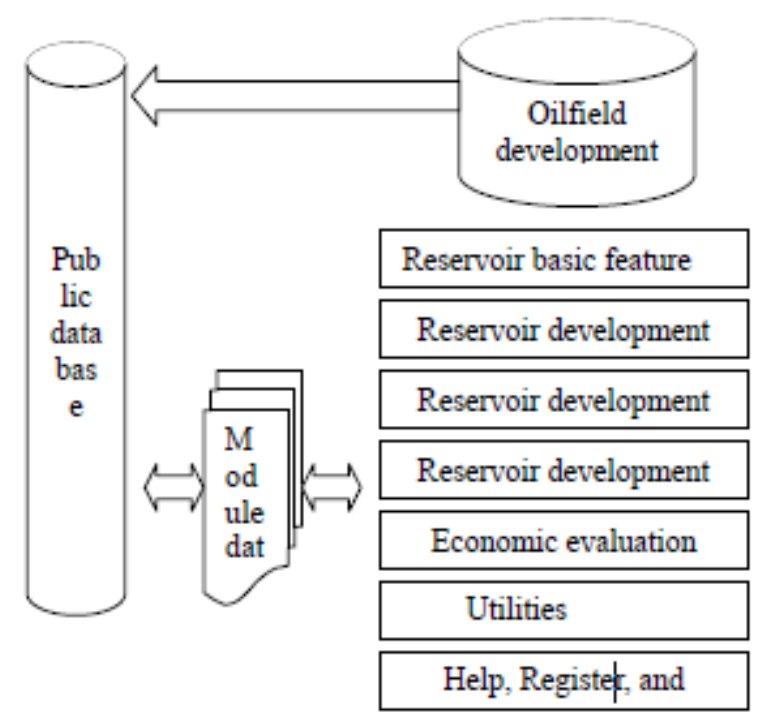

Figure $1 . \quad$ Stucture of res

\section{Module Design Of Software}

There are 6 core modules in the first class menu, among which, the project database and database interface module cover 6 second class menu, including the new project, open project, edit static public data, import local dynamic data, browse local data. The basic reservoir analysis module includes 12 menus including oil petrophysical parameters, formation water petrophysical parameters, gas petrophysical parameters, formation rock petrophysical parameters, relative permeability curve, capillary pressure curve, permeability distribution, variation coefficient, well test analysis, volume methods, material balance methods, and well inclination correction. The technical limit analysis includes 9 menus including well density, injection pressure, bottom flow pressure, injection production ratio, injector producer ratio, injection production pressure, producer inflow, horizontal well productivity, multiple phase vertical flow calculation. The development performance module includes 9 menus including development status, composite development curve, single well production curve, reservoir development status, and injection performance evaluation, water flooding performance, water invasion, input error correction, and layer production splitting. The development forecast module includes 10 menus including rate decline, development index, flow pipe probability, water flooding, recovery factor of different type of reservoir, steam stimulation, steam dynamic analysis, CO2 flooding, oil layer burning, and polymer flooding. The economic evaluation module includes 4 menus including economic evaluation, plan selection and optimization, initial rate of single well, and economic limit of water cut.

\section{REALIZATION AND APPLICATION OF SOFTWARE SYSTEM}

The popular programming techniques are used in the realization of functions based on detailed design, and optimized for the connection function. In accordance to the requirement of software, the software is reformed to accommodate the industrial promotion and utilization.

\section{A. Optimization Of General Function}

The connection with the development database is the source of the data used in the software, and the public database will provide the data sharing.

(1) The data dictionary is utilized to realize the reading interface with the development database: adopting the ADO database to realize the visiting of Oracle database. The ADO is a very simple format to connect with the ODBC, and therefore can use any kind of ODBC data source. It can be used int he SQLServer, Oracle, and Access database, as well as in the Excel, TXT and Image file.

(2) The parallel management coupling the public and module database, so as to realize the one time input and data sharing: the public database is designed to accommodate the requirement of data source and introduction of data used for many calculation modules. This is designed for the requirement of data sharing, and the data used in one module can be accessed by other modules, and can be edited by other modules for convenience. And, the public database can offer the integrated connection and database edit window.

\section{B. Realization Of The Module Procedures}

The current geological software is designed in modules, and the procedure will guide the whole process, and this process will be recorded for the subsequent project study, and once the data is introduced into the system, the whole study process will be completed.

\section{Realization Of Function}

(1)The basic reservoir feature analysis: The basic reservoir analysis module includes 12 menus including oil petrophysical parameters, formation water petrophysical parameters, gas petrophysical parameters, formation rock petrophysical parameters, relative permeability curve, capillary pressure curve, permeability distribution, variation coefficient, well test analysis, volume methods, material balance methods, and well inclination correction[3][4][5].

12 second order menus, 14 third order menus, and totally 23 function modules.

(2)Development technical limits analysis: The technical limit analysis includes 9 menus including well density, injection pressure, bottom flow pressure, injection production ratio, injector producer ratio, injection production pressure, producer inflow, horizontal well productivity, multiple phase vertical flow calculation. 8 second order menus and 9 third order menus.

(3)The development performance module includes 9 menus including development status, composite development curve, single well production curve, reservoir development status, and injection performance evaluation, 
water flooding performance, water invasion, input error correction, and layer production splitting.

(4) The development forecast module includes 10 menus including rate decline, development index, flow pipe probability, water flooding, recovery factor of different type of reservoir, steam stimulation, steam dynamic analysis, CO2 flooding, oil layer burning, and polymer flooding.

(5) The economic evaluation module includes 4 menus including economic evaluation, plan selection and optimization, initial rate of single well, and economic limit of water cut.

\section{Software Coding And Test}

The software system adopts the Windows programming and interface to realize the icon of functions, and the input interface is integrated into the main interface. The data and the image can be displayed simultaneously in the same window, and the date is left, the image right. The ActiveBar is introduced in the Visual Basic programming environment, and all the menus are redesigned for the interface of Windows with 50,000 lines of programming codes.

The test is for the errors correction before the formal utilization, and it is divided into module test, software integrated test and user application test. The software integrated test refers to the comprehensive test on the integrated module in the system, so as to find the problems concerning the connection and interface. The initial test has been accomplished, and the formal software system is further tested by users in Geoscience Research Institute and oil production plants, totally of 110 softwares are installed with good performance.

\section{CONCLUSION}

This paper is based on the data collection, organization, filtering of reservoir engineering methods, and then the adaptability is studied to construct the reservoir engineering core content including basic reservoir features analysis, development technical limit analysis, development performance analysis, development forecast and economic evaluation, it is connected with the development database and then provides the data sharing. It proposes the detailed design oriented on the function realization and procedures. Based on the detailed design, the functions are realized by means of WINDOWS programming and control programming techniques, and the general functions are optimized. The test and application of the software prove that the software can meet the requirement, and can be used in the field.

\section{REFERENCES}

[1] Yuanqian Chen. Reservoir Engineering Calculation Method [M]. Beijing,Petrelum Industry Press, pp. 21-33, 1990

[2] Rucheng Cai,Yang Li and Huanquan Sun. Reservoir Engineering Method and Application[M].DongYing: Petrelum University Press, pp. 15-18, 2002

[3] Qitai Yu. Three Large Remaining Oil Enrichment Region No Spread about Water flooding Reservoir[J].Petroleum Industry Press, pp. 3443, 2000

[4] Xianzhang Tong.Oil Well Production and Reservoir Dynamic Analysis [M]. Beijing,Petrelum Industry Press, pp. 80-89, 1990

[5] Tongluo Qing,Dang Li and Yuanqian Chen.Practical Reservoir Engineering Method[M];Beijing,Petrelum Industry Press, pp. 51-67, 1989. 